Return to the Manage Active Submissions page at http://spie.org/submissions/tasks.aspx and approve or disapprove this submission. Your manuscript will not be published without this approval. Please contact author_help@spie.org with any questions or concerns.

\title{
Application of Firefly Algorithm to the dynamic model updating problem
}

\author{
Faisal Shabbir ${ }^{1}$, Piotr Omenzetter ${ }^{2 *}$ \\ ${ }^{1}$ The University of Engineering and Technology, Taxila, Pakistan \\ ${ }^{2}$ The LRF Centre for Safety and Reliability Engineering, The University of Aberdeen, Aberdeen, UK
}

\begin{abstract}
Model updating can be considered as a branch of optimization problems in which calibration of the finite element (FE) model is undertaken by comparing the modal properties of the actual structure with these of the FE predictions. The attainment of a global solution in a multi dimensional search space is a challenging problem. The nature-inspired algorithms have gained increasing attention in the previous decade for solving such complex optimization problems. This study applies the novel Firefly Algorithm (FA), a global optimization search technique, to a dynamic model updating problem. This is to the authors' best knowledge the first time FA is applied to model updating. The working of FA is inspired by the flashing characteristics of fireflies. Each firefly represents a randomly generated solution which is assigned brightness according to the value of the objective function. The physical structure under consideration is a full scale cable stayed pedestrian bridge with composite bridge deck. Data from dynamic testing of the bridge was used to correlate and update the initial model by using FA. The algorithm aimed at minimizing the difference between the natural frequencies and mode shapes of the structure. The performance of the algorithm is analyzed in finding the optimal solution in a multi dimensional search space. The paper concludes with an investigation of the efficacy of the algorithm in obtaining a reference finite element model which correctly represents the as-built original structure.
\end{abstract}

Keywords: dynamic testing, model updating, firefly algorithm, optimization, cable-stayed bridge, system identification.

\section{INTRODUCTION}

For the design and analysis of civil engineering systems, the finite element (FE) method is widely used. FE models of civil structures are usually based on idealized drawings/designs and estimates of material properties, structural geometry and boundary and connectivity conditions, which may not truly reflect the exact behavior of the as-built system. Field tests can be performed on the full-scale structures to validate the FE models. The dynamic behavior of structures under external excitation is of major interest to structural engineers, as the experimental responses (such as modal frequencies, damping and shapes) are related to physical properties of the system (mass, stiffness and energy dissipation). Full-scale, in-situ dynamic experimental investigations of civil engineering structures have gained increasing popularity as they enable identification of the actual dynamic properties useful in performance and health monitoring as well as for maintenance. Information from such experiments can more effectively be used by correlating the experimental outcomes with analytical models. Significant differences in the dynamic behavior of FE models and as-built systems have been noted by various researchers ${ }^{1-3}$ and numerous studies focusing on damage detection and estimation have been carried out using vibration data, 5 . A method often used for such correlation is model updating where optimal values of the parameters of an analytical model, such as stiffness, mass and/or boundary conditions, are searched for in order to match analytical responses with those identified experimentally. As the number of experimental measurements is usually much smaller than the number of uncertain parameters, and, consequently, not all uncertain parameters are selected for model updating, different local minima may exist in the solution space. Experimental noise further exacerbates the problem. The attainment of the global minimum in multi-dimensional search space is a challenging optimization problem.

The traditional model updating approaches often use the sensitivity method (SM) which gives a single updated solution ${ }^{2}$ but has a tendency to converge to local minima ${ }^{6}$. Although model updating using SM-based optimization has been well

* piotr.omenzetter@abdn.ac.uk; phone 441224-272529; fax 44-1224-272497; www.abdn.ac.uk 
Return to the Manage Active Submissions page at http://spie.org/submissions/tasks.aspx and approve or disapprove this submission. Your manuscript will not be published without this approval. Please contact author_help@spie.org with any questions or concerns.

documented, the application of global optimization algorithms (GOAs) in this field have received limited attention. However, they can explore the search space more widely and can therefore avoid being trapped in local minima.

Most recent studies which report GOA-based model updating are for damage detection of laboratory-scaled structures. Genetic Algorithm (GA) was used ${ }^{7}$ for assessment and damage detection of a simulated beam structure and an experimental beam structure. Multiple damage scenarios were studied along with the effect of different noise levels on a simulated beam structure. In another study ${ }^{8}$, both Simulated Annealing (SA) and GA were investigated to update a numerical model of a cantilever beam and an experimental wing plate structure. Saada et al. ${ }^{9}$ used Particle Swarm Optimization for model updating of a beam structure. However, it can be noticed that most research efforts using GOAs have been made towards damage detection and assessment of simulated structures or simple laboratory scale structures and updating of full scale structures still remains a challenging and relatively unexplored topic in the applications of GOAs.

A recent development in meta heuristic algorithms is Firefly Algorithm (FA) proposed by Yang ${ }^{10}$. The FA is inspired by the flashing characteristics of fireflies. In this paper, FA is explored for dynamic FE model updating of a dynamically tested full scale cable stayed pedestrian bridge.

In the sections to follow, the theories related to the FA and objective functions in model updating are detailed. Then a description of the full scale bridge and its dynamic testing results are presented. FA was then applied for model updating of a numerical model of the full scale bridge to match the actual dynamic testing results with the FE model counterparts. Finally, the updated results obtained are discussed and physically justified.

\section{THEORY}

\subsection{Firefly Algorithm}

$\mathrm{FA}^{11}$ is a population-based stochastic optimization method that tries to improve the solution iteratively with respect to a given measure of quality. The concept of FA was developed based on the flashing behavior of fireflies. The flashing light of fireflies is an example bioluminescence, and is used by the fireflies to communicate and attract prey. The three idealized rule followed by the algorithm are:

1. Fireflies are unisex and they are attracted to each other regardless of sex.

2. Attractiveness depends upon the brightness, i.e. for a couple the less bright fly will be attracted to the brighter one. Therefore attractiveness is proportional to brightness and is assumed to decrease as the distance between the fireflies increases. The firefly will move randomly in the case if there is no brighter firefly in the population than herself.

3. Brightness of a particular firefly depends on the shape of the objective function.

In the FA, the two important parameters are the variation of light intensity and attractiveness formulation. In simple sense, it can be assumed that the attractiveness is dependent on brightness which is associated with the objective function value.

As the intensity of light decreases with the distance from the source, its variation should also be a monotonically decreasing function. Attractiveness also behaves in a similar manner. The following Gaussian form can be used as an approximation for representing the combined effect of both the inverse square law and absorption:

$$
I(r)=I_{o} e^{-\gamma r^{2}}
$$

where $I_{o}$ is the original light intensity, $r$ is the distance and $\gamma$ is the absorption coefficient which can be taken as constant. As the firefly attractiveness is directly proportional to the intensity of light, the attractiveness $\beta$ can be defined as:

$$
\beta(r)=\beta_{o} e^{-\gamma r^{2}}
$$

where $\beta_{o}$ is attractiveness at $r=0$. The distance between the fireflies can be calculated using the Euclidian distance.

\section{7 - 33 V. 1 (p.2 of 8) / Color: No / Format: A4 / Date: 3/5/2015 3:02:52 PM}


Return to the Manage Active Submissions page at http://spie.org/submissions/tasks.aspx and approve or disapprove this submission. Your manuscript will not be published without this approval. Please contact author_help@spie.org with any questions or concerns.

The movement of firefly $k$ to a brighter firefly $l$ can now be determined by:

$$
x_{k}=x_{k}+\beta_{o} e^{-\gamma r^{2}}\left(x_{l}-x_{k}\right)+\alpha \varepsilon_{k}
$$

where $\alpha$ is the randomization parameter and $\varepsilon_{k}$ is a random number chosen from the Gaussian distribution.

\subsection{Objective function for model updating}

A combined objective function related to the frequencies and model assurance criterion ${ }^{12}$ (MAC) is used in this study. The relative error between the experimental and analytical frequencies is:

$$
\Pi_{1}=\sum_{i=1}^{n}\left[\left(\omega_{a, i}-\omega_{e, i}\right) / \omega_{e, i}\right]^{2}
$$

where $\omega$ represents modal frequency, subscripts $a$ and $e$ refer to the analytical and experimental values, respectively, and $n$ is the total number of modes to be updated. The second objective function is related to the difference in mode shapes and can be defined in terms of MAC as:

$$
\Pi_{2}=\sum_{i=1}^{n}\left(1-\sqrt{M A C_{i}}\right)^{2} / M A C_{i}
$$

Combined objective function $\Pi$ is defined as follows:

$$
\Pi=\Pi_{1}+\Pi_{2}
$$

\section{DESCRIPTION OF THE BRIDGE, FE MODEL AND DYNAMIC TESTING}

The full scale bridge under study is a $59,500 \mathrm{~mm}$ long cable stayed footbridge with two symmetrical spans separated by a central A-shaped pylon over a four-lane motorway. The bridge shown in Figure 1 and basic dimensions are provided in Figure 2. The bridge deck is a trapezoidal steel girder with cast in-situ concrete slab of thickness $130 \mathrm{~mm}$. The deck is continuous over the entire span and is supported by a total of six pairs of stay cables with approx. $8 \mathrm{~m}$ centre to centre distances. Different post-tension forces have been applied to each pair of cables ranging from $55 \mathrm{kN}$ to $95 \mathrm{kN}$ as shown in Figure 2. The cables were connected to the top of the pylon, which is composed of two steel I-sections joined with cross bracing that also supports the deck. The size of the pylon I-section is $400 \mathrm{WC} 328^{13}$ and the diameter of the cables used is $32 \mathrm{~mm}$. A closed rectangular pipe having a section of $250 \times 150 \times 9 \mathrm{~mm}$ also runs on both sides of the bridge deck and this encloses two $100 \mathrm{~mm}$ ducts for service pipes with surrounding void spaces grouted with cement. A railing, which has a total height of $1400 \mathrm{~mm}$, is provided on both sides of the bridge. The panels of railings are disconnected from each other at every $8 \mathrm{~m}$ centre to centre. The bridge is supported on pivot type steel bearings at the central pylon and sliding steel-concrete bearings at the abutments. The sliding bearings at the support were provided to accommodate creep, shrinkage and temperature deformations, and to allow the bridge to move longitudinally in the event of a strong seismic excitation. The bridge is supported by two concrete piles at each abutment and ten concrete piles at the central pylon through a pile cap on their top.

The main elements to be modeled in case of cable stayed bridges are the deck, pylon, cables and connections of cables and deck. A reasonable representation of bridge deck for box girder sections can be achieved by using beam elements with rigid links joining the cable elements with deck elements ${ }^{14,}{ }^{15}$. In this research, the bridge was modeled in SAP $2000^{16}$. The modulus of elasticity of steel was taken as $2.00 \times 10^{11} \mathrm{~N} / \mathrm{m}^{2}$ and of concrete it was taken as $2.80 \times 10^{10} \mathrm{~N} / \mathrm{m}^{2}$. The deck and pylon were modeled using beam type FEs. The beam was discretized in to 48 elements, whereas pylon was discretized into 40 elements. The cables were modeled using catenary elements provided in SAP2000 and were discretized into four elements for each cable. Further discretization of these elements did not appreciably affect the natural frequencies and only resulted in an increase in the computational cost. An initial non-linear static analysis was performed to account for the geometric non-linearity caused by the cable sag and that was followed by a linear dynamic analysis to obtain natural frequencies and mode shapes. A linear analysis that uses stiffness from the end of non-linear static analysis for cable stayed structures has been demonstrated to provide accurate results ${ }^{17}$. 
Return to the Manage Active Submissions page at http://spie.org/submissions/tasks.aspx and approve or disapprove this submission. Your manuscript will not be published without this approval. Please contact author_help@spie.org with any questions or concerns.

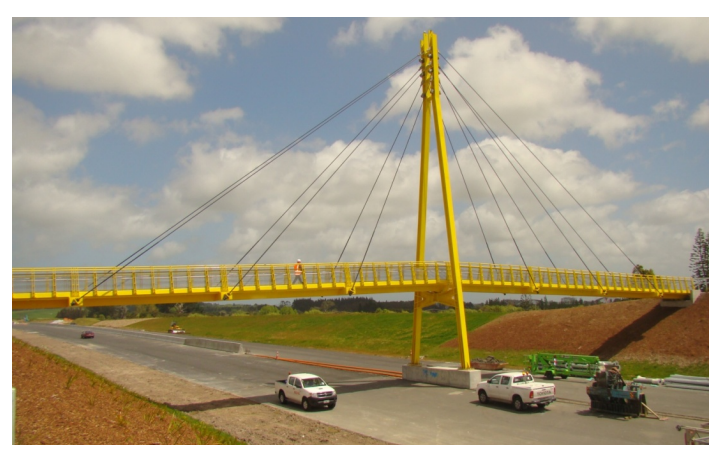

Figure 1 Full scale cable stayed bridge

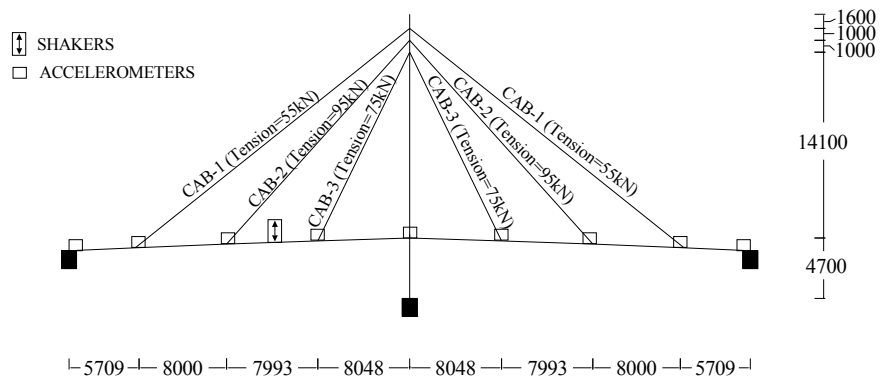

Figure 2 Basic dimensions (in $\mathrm{mm}$ ) of the bridge, cable forces, and shaker and accelerometer locations

Experimental work was carried out using 15 uni-axial Honeywell QA 750 accelerometers to measure structural response, 3 uni-axial Crossbow MEMS accelerometers to measure shaker input force and a desktop computer fitted with NI DAQ 9203 data acquisition card. Data was collected at a sampling rate of $200 \mathrm{~Hz}$. Three APS Electroseis Model 400 shakers $^{18}$ were used in synchronized mode to impart a combined excitation force of up to $1.2 \mathrm{kN}$ to the structure.

Full scale tests can be conducted by output only (no measured force) or input-output (measured force) methods. The cable-stayed bridge under study has been tested using both of these methods. The output only test was conducted using jumping to establish the initial estimation of the natural frequencies of the bridge. Two people jumped several times on the bridge in unison to excite the structure and thereafter the bridge was allowed to freely vibrate for two minutes. This was done to establish the range of excitation frequencies for subsequent forced vibration tests. The forced vibration test was then conducted using three dynamically synchronized shakers and their input force was measured using accelerometers mounted on the armature. A sweep sine excitation frequency ranging from 1 to $15 \mathrm{~Hz}$ with the total sweep duration of 466.7 seconds was adopted to adequately excite the structure. The shakers were located away from the deck center line to excite both the vertical and torsional modes. To obtain the natural frequencies in the horizontal direction, the shakers were tilted at 90 degrees. The location of the accelerometers and shakers is shown in Figure 2.

The Numerical Algorithm for Subspace State-Space System Identification (N4SID) ${ }^{19}$, a powerful technique for modal identification in the time domain, is used for system identification in this study. To eliminate spurious results, stability diagrams are employed. As the system order increases, the structural modes identified by the algorithm should remain stable $^{20}$. Stability tolerances were selected based on the experience and data quality. A tolerance of $1 \%$ for frequency for two subsequent model orders and a value above 0.9 for MAC were selected as the mode stability criteria in this study.

Table 1 summarizes the natural frequencies identified by the N4SID method, and also shows the errors between the experimental frequencies and those identified by the initial FE model. It has been found that the frequencies obtained from the initial FE model differ from the experimental frequencies by as much as $8.6 \%$. However, MAC values are greater than 0.96 showing that there is a good correlation between the observed and initial FE model mode shapes.

\section{MODEL UPDATING}

In model updating, dynamic measurements such as natural frequencies and mode shapes are normally correlated with their FE model counterparts to calibrate the FE model. There is a degree of uncertainty in the assessment of the actual properties of the materials used in the full scale structure as well as the most realistic representation of the element stiffness in the initial FE model. The challenge of finding a set of suitable parameters having physical justification necessitates the use of physically significant updating parameters and suitable optimization tools. 
Return to the Manage Active Submissions page at http://spie.org/submissions/tasks.aspx and approve or disapprove this submission. Your manuscript will not be published without this approval. Please contact author_help@spie.org with any questions or concerns.

Table 1 Natural frequencies by N4SID, initial FE model, and errors between initial FE model and experimental results

\begin{tabular}{|ccccc|}
\hline & \multicolumn{3}{c|}{ Frequency } & \\
\cline { 2 - 4 } Mode No. & Experiment by N4SID & Initial FE model & Error & \\
\hline 1 & $\mathbf{( H z )}$ & $(\mathbf{H z})$ & $\mathbf{( \% )}$ & MAC \\
2 & 1.64 & 1.66 & 1.2 & 0.999 \\
3 & 1.90 & 1.88 & -1.1 & 0.995 \\
4 & 3.69 & 3.88 & 5.2 & 0.999 \\
5 & 4.86 & 5.28 & 8.6 & 0.999 \\
6 & 5.33 & 5.45 & 2.3 & 0.993 \\
7 & 6.31 & 6.79 & 7.6 & 0.990 \\
8 & 7.42 & 7.76 & 4.6 & 0.980 \\
& 8.32 & 8.66 & 4.1 & 0.993 \\
\hline
\end{tabular}

The selection of parameters for model updating is a crucial step. Only those parameters should be selected to which the responses are sensitive and whose values are uncertain in the initial model. In this study, only a few parameters were selected based on a sensitivity analysis conducted to find out the influential parameters. Possible parameters for this study include cable tensions, cable stiffness, stiffnesses of the deck and stiffnesses of the central pylon and boundary conditions. The final selected parameters based on the sensitivity analysis (see Figure 3 ) and engineering insight were deck flexural stiffness for vertical $\left(K_{y, \text { Deck }}\right)$ and horizontal $\left(K_{x, \text { Deck }}\right)$ bending, deck torsional stiffness $\left(K_{t, \text { Deck }}\right)$, axial stiffness of all cables $\left(K_{\text {Cable }}\right)$, cable tension for Cab-1 $\left(T_{C a b-1}\right)$ and stiffness of bearings $\left(K_{\text {bearing }}\right)$. It can be noted from Figure 3 that the sensitivity of $K_{t \text { Deck }}$ and $K_{\text {bearing }}$ effects mode 8 in a similar manner. Therefore a regularization terms was introduced in Equation 6 as:

$$
\Pi=\Pi_{1}+\Pi_{2}+0.0005 \times\left|\frac{K_{t, \text { Deck }, i}}{K_{\text {bearing }, i}}-\frac{K_{t, \text { Deck }, 0}}{K_{\text {bearing }, 0}}\right|
$$

where the coefficient 0.0005 represents the tradeoff between the regularization term and original objective function; a higher value tends to fix the two parameters $K_{t, \text { Deck }}$ and $K_{\text {bearing }}$ to change together and a lower value allows them to move away from each other during updating. This value has been selected so that the ratio of the two parameters approximately remains within $\pm 20 \%$. The subscript ' $i$ ' represents values of these parameters in the current iteration step and subscript ' 0 ' represents the values of these parameters corresponding to the initial FE model.

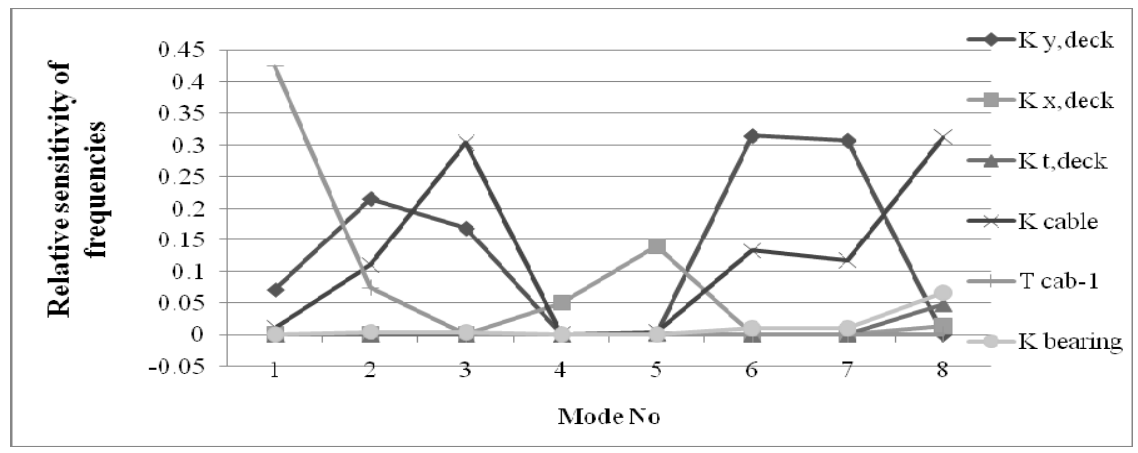

Figure 3 Relative sensitivity of frequencies 


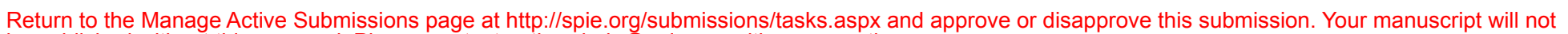
be published without this approval. Please contact author_help@spie.org with any questions or concerns.

It is hard to estimate the variation bounds of the parameters during model updating and this is normally done using engineering judgment. Different bounds have been used in various studies ${ }^{21}$. From the frequency errors in Table 1 , it can be concluded that the initial FE model overestimates the stiffness, therefore the lower bound has been selected as $-40 \%$, and the upper bound has been selected as $+30 \%$ for all the parameters.

A population of 20 fireflies was used and the maximum number of generations was set to 100 . The other parameters were set to $\alpha=0.25, \beta_{o}=0.1$ and $\gamma=1$. The algorithm was terminated when one of the two criteria i.e. the minimum objective function value of 0.0020 or the maximum number of iterations of 100 was satisfied.

Model updating using FA was attempted by using the six aforementioned unknown parameters related to the stiffness of the bridge. The updated solutions in the form of the ratios of the updated values to the initial values are given in Table 2. The table also indicates the final value of the objective function obtained.

Table 2 Ratio of updated to initial stiffness values obtained by FA and final objective function value.

\begin{tabular}{|ccccccc|}
\hline \multicolumn{5}{|c|}{ Ratio of updated to initial stiffness by FA } & \multirow{2}{*}{ Final obj func. value } \\
\cline { 1 - 6 } $\mathbf{K}_{\mathbf{y}, \text { deck }}$ & $\mathbf{K}_{\mathbf{x}, \text { deck }}$ & $\mathbf{K}_{\mathrm{t} \text {,deck }}$ & $\mathbf{K}_{\text {cable }}$ & $\mathbf{T}_{\text {Cab-1 }}$ & $\mathbf{K}_{\text {bearing }}$ & \\
\hline 0.843 & 0.853 & 0.952 & 0.908 & 1.111 & 0.922 & 0.0024 \\
\hline
\end{tabular}

The updating results found by FA (Table 2) are discussed now. The updated frequencies and their errors compared to the experimental results, and updated MAC values between the experimental and model results are shown in Table 3. It has been found that FA has given improved results after model updating as compared to the initial frequency estimates. The frequencies and their errors for the updated FE model and experimental results show that maximum frequency difference of $8.6 \%$ in the initial FE model (Table 2) has been reduced to $2.4 \%$ in the updated model. The updated MAC values shown in Table 3 indicate that MACs have either been improved or at least not degraded in the process.

The updated parameters should be physically meaningful; otherwise it is difficult to justify the results with respect to the actual structure. The vertical stiffness of the bridge deck has decreased by $14.7 \%$, the horizontal stiffness by $18.2 \%$ and the torsional stiffness by $4.8 \%$ respectively. This could be mainly attributed to the fact that the initial model took the cast in-situ concrete slab as fully composite with the steel girder. The updated results revealed that there is only partial composite action between the slab and the steel girder resulting in lower stiffness of the whole deck. The consistent decrease in all the parameters related to the deck stiffness supports this conclusion. The cable axial stiffness has been reduced by $9.2 \%$ which can be attributed to many factors such as loosening of cables, joint stiffness and, breakage of cable tendons. On the other hand, the increase in cable tension $T_{C a b-1}$ by $11.1 \%$ shows that the post-tension forces in the far most cable stays are more than the designed value of $55 \mathrm{kN}$. Also decrease is the combined bearing stiffness $K_{\text {bearing }}$ of $7.8 \%$ could be attributed to the decrease in the modulus of elasticity of rubber bearings.

Table 3 Updated frequencies and MACs

\begin{tabular}{|ccccc|}
\hline Mode No. & \multicolumn{3}{c}{ Frequencies } & Updated MAC \\
\cline { 2 - 4 } & $\begin{array}{c}\text { Experiment } \\
(\mathbf{H z})\end{array}$ & $\begin{array}{c}\text { Updated FE model } \\
\mathbf{( H z )}\end{array}$ & $\begin{array}{c}\text { Error } \\
\mathbf{( \% )}\end{array}$ & \\
\hline 1 & 1.64 & 1.68 & 2.3 & 0.999 \\
2 & 1.89 & 1.86 & -2.4 & 0.996 \\
3 & 3.69 & 3.68 & -0.5 & 0.999 \\
4 & 4.86 & 4.98 & 2.4 & 0.990 \\
5 & 5.33 & 5.28 & -1.1 & 0.987 \\
6 & 6.31 & 6.38 & 1.1 & 1.000 \\
7 & 7.42 & 7.30 & -1.7 & 0.992 \\
8 & 8.32 & 8.36 & 0.5 & 0.993 \\
\hline
\end{tabular}


Return to the Manage Active Submissions page at http://spie.org/submissions/tasks.aspx and approve or disapprove this submission. Your manuscript will not be published without this approval. Please contact author_help@spie.org with any questions or concerns.

\section{CONCLUSIONS}

A systematic study of updating of a cable-stayed pedestrian bridge model employing FA is presented in this paper. Following are the conclusions drawn from this study:

1. Five vertical modes, two horizontal modes and one torsional mode of vibration have been successfully identified for the cable-stayed bridge.

2. FA-based model updating has been successful in reducing the frequency differences between the initial FE model and the experimental results.

3. The final updated FE model of the bridge is able to produce good agreement with the experimental results still preserving the physical meaning of updated parameters.

4. The results indicate that the slab is not fully composite with the steel girder and the tensions in some cables are more than assumed in design.

5. Further research is recommended to improve the convergence behaviour with optimal parameters of FA for finite element model updating problems.

\section{REFERENCES}

[1] Schlune, H., Plos, M. and Gylltoft, K., "Improved bridge evaluation through finite element model updating using static and dynamic measurements," Engineering Structures 31 (7), 1477-1485 (2009).

[2] Brownjohn, J. M. W., Xia, P. Q., Hao, H. and Xia, Y., "Civil structure condition assessment by FE model updating: Methodology and case studies," Finite Elements in Analysis and Design 37 (10), 761-775 (2001).

[3] Friswell, M. I. and Mottershead, J. E., Finite element model updating in structural dynamics, Kluwer Academic Publishers, Netherlands, (1995).

[4] Hu, N., Wang, X., Fukunaga, H., Yao, Z. H., Zhang, H. X. and Wu, Z. S., "Damage assessment of structures using modal test data," International Journal of Solids and Structures 38 (18), 3111-3126 (2001).

[5] Hester, D. and González, A., "A wavelet-based damage detection algorithm based on bridge acceleration response to a vehicle," Mechanical Systems and Signal Processing 28 (0), 145-166 (2012).

[6] Deb, K., Optimization for engineering design: Algorithms and examples, Prentice-Hall of India, New Delhi, (1998).

[7] Perera, R. and Torres, R., "Structural damage detection via modal data with genetic algorithms," Journal of Structural Engineering, ASCE 132 (9), 1491-1501 (2006).

[8] Levin, R. I. and Lieven, N. A. J., "Dynamic finite element model updating using simulated annealing and genetic algorithms," Mechanical Systems and Signal Processing 12 (1), 91-120 (1998).

[9] Saada, M. M., Arafa, M. H. and Nassef, A. O., "Finite element model updating approach to damage identification in beams using particle swarm optimization," Proc. 34th Design Automation Conference, ASME, 522-531 (2008).

[10] Yang, X.-S., "Firefly algorithms for multimodal optimization," in Stochastic algorithms: Foundations and applications, O. Watanabe and T. Zeugmann, Editors, Springer Berlin Heidelberg, 169-178 (2009).

[11] Kennedy, J. and Eberhart, R., "Particle swarm optimization," Proc. IEEE International Conference on Neural Networks, 1942-1948 (1995).

[12] Möller, P. W. and Friberg, O., "Updating large finite element models in structural dynamics," AIAA Journal 36 (10), 1861-1868 (1998).

[13] AS/NZS, As/nzs 3679 Part-1 Structural steel hot rolled bars and sections, Standards Australia, Sydney and Standards New Zealand, Wellington, (1996).

[14] Ren, W. X. and Peng, X. L., "Baseline finite element modeling of a large span cable-stayed bridge through field ambient vibration tests," Computers and Structures 83 (8-9), 536-550 (2005).

[15] Chang, C. C., Chang, T. Y. P. and Zhang, Q. W., "Ambient vibration of long-span cable-stayed bridge," Journal of Bridge Engineering, ASCE 6 (1), 46-53 (2001).

[16] SAP2000, Structural analysis program, Computers and Structures, CA. 2009.

[17] Abdel-Ghaffar, A. M. and Khalifa, M. A., "Importance of cable vibration in dynamics of cable-stayed bridges," Journal of Engineering Mechanics, ASCE 117 (11), 2571-2589 (1991). 
Return to the Manage Active Submissions page at http://spie.org/submissions/tasks.aspx and approve or disapprove this submission. Your manuscript will not be published without this approval. Please contact author_help@spie.org with any questions or concerns.

[18] APSDynamics, Dynamic electroseis shaker model 400. http://www.Apsdynamics.Com/ 2012.

[19] Van Overschee, P. and De Moor, B., Subspace identification for the linear systems: Theory-implementationapplications, Kluwer Academic Publishers, Netherlands, (1996).

[20] Bodeux, J. B. and Golinval, J. C., "Application of ARMAV models to the identification and damage detection of mechanical and civil engineering structures," Smart Materials and Structures 10 479-489 (2001).

[21] Jaishi, B. and Ren, W. X., "Structural finite element model updating using ambient vibration test results," Journal of Structural Engineering, ASCE 131 (4), 617-628 (2005). 